PROCEEDINGS OF THE

AMERICAN MATHEMATICAL SOCIETY

Volume 138, Number 4, April 2010, Pages 1447-1451

S 0002-9939(09)10051-5

Article electronically published on December 10, 2009

\title{
LENZ-BARLOTTI I.4 PERSPECTIVITY GROUPS ARE ABELIAN
}

\author{
ROBERT A. LIEBLER AND ELIZABETH SCOTT-JANDA
}

(Communicated by Jonathan I. Hall)

\begin{abstract}
We extend a 1972 result of Kantor and Pankin and give a new elementary proof of the assertion in the title for projective planes of arbitrary order. The main tool appears in the very first book on group theory by Jordan in 1870 .
\end{abstract}

The Lenz-Barlotti classification of projective planes is based on the possible configurations of point-line pairs for which the Desargues theorem holds. The Desargues theorem holds for such a pair $(\mathrm{p}, \mathrm{l})$ if and only if the plane admits a full group of perspectivities with center $\mathrm{p}$ and axis I. By definition, such a perspectivity group fixes all points on $\mathrm{I}$, all lines on $\mathrm{p}$ and is maximally transitive consistent with these conditions. The Lenz-Barlotti figure of a projective plane $\Pi$ is the set of point-line pairs for which $\Pi$ is $(p, I)$-transitive and determines the Lenz-Barlotti class of $\Pi$. The plane $\Pi$ is of class I.4 (respectively I.3) if its Lenz-Barlotti figure consists of the three non-incident point-line pairs of a triangle (respectively two of these pairs). These classes are two of the five for which existence questions remain open [1] The purpose of this paper is to give a new elementary proof of:

Theorem 1. Let $\Pi$ be a projective plane of Lenz-Barlotti type I.4. Then its three transitive perspectivity groups are isomorphic and abelian.

Kantor and Pankin [6] prove this when $\Pi$ is finite, using deep results of Suzuki regarding finite groups with subgroups that partition the group's non-identity elements.

For the group $G$, the right and left regular representations are homomorphisms from $G$ to $\operatorname{Sym}(G)$ (the group of permutations of elements of $G$ ) defined by: $h^{\lambda(g)}=$ $g^{-1} h ; h^{\rho(g)}=h g, g, h \in G$. Our main tool is the elementary interplay between $\lambda(G)$ and $\rho(G)$ [2, p. 86]:

Theorem 2 (Jordan [4 p. 60]). If $\sigma \in \operatorname{Sym}(G)$ commutes with $\rho(g)$ for all $g \in G$, then $\sigma=\lambda(h)$ for some $h \in G$. This holds symmetrically with $\lambda$ and $\rho$ reversed.

Use the permutation group notation of Wielandt 8. In particular, suppose the group $G$ acts on the set $\Omega$, so $\Omega$ is a $G$-space. For $\omega \in \Omega, G_{\omega}:=\left\{g \in G: \omega^{g}=\omega\right\}$.

Suppose $G$ acts transitively on $\Omega$. Then the map $\Omega \rightarrow G_{\omega} \backslash G:=\left\{G_{\omega} g: g \in G\right\}$ given by $\omega^{g} \mapsto\left(G_{\omega}\right) g$ is a $G$-space isomorphism, and the set $G_{\omega} \backslash G$ of right $G_{\omega}$

Received by the editors July 30, 2008, and, in revised form, April 28, 2009.

2000 Mathematics Subject Classification. Primary 51-00, 05-00.

${ }^{1}$ Existence of finite planes in either class is open. Naumann 7 constructs infinite planes in class I.4.

(C)2009 American Mathematical Society Reverts to public domain 28 years from publication 
cosets in $G$ is an internal realization of $\Omega$. Since $x^{-1} G_{\omega} x=G_{\omega^{x}}$, different choices for $\omega \in \Omega$ lead to isomorphic $G$-spaces.

The group $G$ is an automorphism group of the incidence structure $(X, Y, I)$ if $X$ and $Y$ are $G$-spaces and the induced action of $G$ on $X \times Y$ leaves $I$ invariant. A coset geometry associated with the action of $G$ on $I$ is an isomorphic incidence structure consisting entirely of internal transitive $G$-spaces and $G$-invariant relations between them. When $G$ acts transitively on both $X$ and $Y$, an associated coset geometry only requires starters $x \in X, y \in Y$. Then the $G$-orbit $(x, y)^{G} \subset X \times Y$ is uniquely associated with the $G$-orbit $\left\{\left(G_{x} g, G_{y} g\right) \mid g \in G\right\} \subset G_{x} \backslash G \times G_{y} \backslash G$. We say such a coset geometry is synchronized if $(x, y) \in I$.

By building coset geometries for multiple incidence structures that admit the same abstract group, one can reveal subtle relationships between known combinatorial structures. When an incidence structure has a sufficiently rich automorphism group $G$, Theorem 2 allows the incidence relation to be expressed in terms of $\lambda: G \rightarrow \operatorname{Sym}(G)$ as we do in section 2. Under the assumptions of Theorem 1, we show that another automorphism group $Z$ is forced into $\operatorname{Im}(\lambda)$ and there results a certain equality (equation (3)). The proof of Theorem 1 is completed by a combinatorial interpretation of this equality using $\rho(G)$.

\section{Geometric preliminaries}

The projective plane $\Pi=\left(P^{*}, L^{*}, F^{*}\right)$ has points $P^{*}$, lines $L^{*}$ and incident point-line pairs (flags) $F^{*}$. Lowercase sans serif font is used for points and lines exclusively. The line on points $x$ and $y$ is written $x y$, and the juxtaposition of two line labels denotes their point of intersection.

Suppose that $\Pi$ is of Lenz-Barlotti class at least I.3. This means there are three non-collinear points $\mathrm{x}, \mathrm{y}, \mathrm{z}$ so that there is a full $(\mathrm{x}, \mathrm{yz})$-perspectivity group $X$ as well as a full $(\mathrm{y}, \mathrm{xz})$-perspectivity group $Y$. Set $G:=\langle X, Y\rangle$ and observe that there are seven $G$-orbits on points which we label as indicated in the figure below.

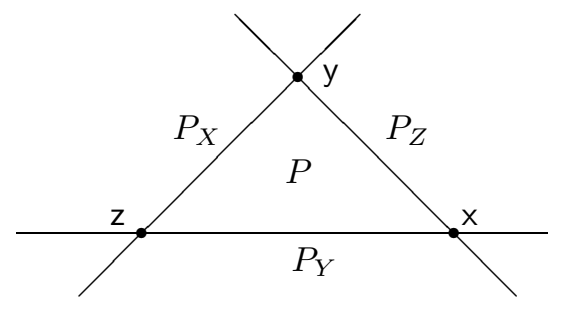

For example, $P_{X}$ consists of the points of yz not in $\{\mathrm{y}, \mathrm{z}\} . P_{Y}$ and $P_{Z}$ are defined analogously, and $P$ consists of all points off the triangle $x$ y $z$. We call elements of $P$ ordinary. Points of the triangle which are not vertices are called axial, and they fall into the sets $P_{X}, P_{Y}$, and $P_{Z}$. Ordinary and axial lines are defined dually.

Lemma 1. The groups $X$ and $Y$ commute and are normal in $G$. Moreover, $G$ is the internal direct product of $X$ and $Y$ and acts faithfully and regularly on both $P$ and $L$.

Proof. The first claim is a consequence of the fact that $X$ is the kernel of the action of $G$ on $P_{X}$ and it acts regularly (sharply transitively) and faithfully on $P_{Y}$, while $Y$ is the kernel of the action of $G$ on $P_{Y}$ and acts regularly while acting faithfully on $P_{X}$. Therefore, $x \in X$ and $y \in Y$ commute on $P_{X} \cup P_{Y}$. 
The ordinary point $\mathrm{p}$ is uniquely the intersection of the axial line $\mathrm{px}$ and $\mathrm{py}$, so the images of the axial points $(\mathrm{px})(\mathrm{yz}) \in P_{X}$ and $(\mathrm{py})(\mathrm{xz}) \in P_{Y}$ uniquely determine $\mathrm{p}^{x y}$. The transitivity of $G$ on $P$ therefore follows geometrically from its transitivity on $P_{X} \times P_{Y}$.

The claim for $L$ follows from the above argument applied to the dual plane.

Lemma 2 (Hughes [3]). The groups $X$ and $Y$ are isomorphic. If these groups are abelian, then the kernel of the action of $G$ on $P_{Z}$ is $(\mathrm{z}, \mathrm{xy})$-transitive and $\Pi$ is of type I.4.

Proof. The groups $X$ and $Y$ both act regularly on $P_{Z}$ and commute by Lemma 1 . Therefore, their action on $P_{Z}$ is just as in Theorem 2, and they act as the left and right regular representations of the same group. This establishes the first claim. Suppose $X \cong Y$ is abelian. Then Lemma 1 implies that $G=\langle X, Y\rangle$ is also abelian. Take $\mathrm{w}$ to be any point on $\mathrm{xy}$. Then each axial point on $\mathrm{xy}$ has the form $\mathrm{w}^{x}$ for some $x \in X$, and so the stabilizer is $G_{\mathrm{w}^{x}}=x^{-1} G_{\mathrm{w}} x=G_{\mathrm{w}}$, as $G$ is abelian. This implies that $G_{\mathrm{w}}$ is $(\mathrm{z}, \mathrm{xy})$-transitive.

There are three partitions $\pi_{X}, \pi_{Y}$, and $\pi_{Z}$ of the ordinary points $P$ with the property that two points are in the same part of one of these partitions if and only if they determine an axial line on $\mathrm{x}, \mathrm{y}$ or $\mathrm{z}$, respectively. There is also a dual partition of lines, and together with the ordinary incidence structure $\left(P, L, F:=F^{*} \cap P \times L\right)$ they uniquely determine all incidences for the plane $\Pi$. Thus $\Pi$ is uniquely determined by the incidence relation between the ordinary points and ordinary lines [1].

\section{The ORDINARY INCIDENCE RELATION COSET GEOMETRY}

In this section we show that the incidence relation between ordinary points and ordinary lines appears in the internal coset geometry as the left regular representation of a subset $\Delta$ of $G$. When $\Pi$ is finite, the set $\Delta$ is called a neo-difference set [1.

We are exclusively concerned with collineations of $\Pi$ that fix each of $\mathrm{x}, \mathrm{y}, \mathrm{z}$. Call this group $A$, and note that it normalizes $G$. In order to build an $A$-invariant synchronized coset geometry from $G$ for the ordinary incidence structure, we specify a seed ordinary flag $(\mathrm{p}, \mathrm{I})$, and from it we obtain the ordinary point seed $\mathrm{p} \in P$ and the ordinary line seed $\mathrm{I} \in L$.

Now $H:=G_{\mathrm{pz}}$ acts transitively on the ordinary points of $\mathrm{pz}$. If it were to act trivially on points of xy as well, then the kernel of the action of $G$ on $P_{Z}$ would be $(z, x y)$-transitive, and $\Pi$ would be of type (at least) $I .4$ as in Lemma 2 . Therefore, we assume without loss of generality that there is an axial point $w$ on $x y$ such that $\mathrm{w}^{h} \neq \mathrm{w}$ for some $h \in H$ (see Figure 1 in the next section) and take I:= pw as the line of the seed flag.

Having specified a $G$-coset geometry for the incidence relation between ordinary points and lines, we are now in a position to express this incidence relation in terms of $G$ alone. For $C, D \in\{P, L\}$ and $S \subseteq C \times D$, define

$$
R_{S}:=\left\{(g, h) \in G \times G:\left((\text { seed from } C)^{g},(\text { seed from } D)^{h}\right) \in S\right\} \subseteq G \times G .
$$

Extend this notation to permutations $\pi \in \operatorname{Sym}(G)$ by defining $R_{\pi}:=\left\{\left(g, g^{\pi}\right): g \in\right.$ $G\}$. In particular, if $t \in G$, then

$$
R_{\rho(t)}=\{(g, g t): g \in G\} \text { and } R_{\lambda(t)}=\left\{\left(g, t^{-1} g\right): g \in G\right\} .
$$


For $a \in A$, the associated ordinary point permutation, $a \mid P$, and the associated ordinary line permutation, $a \mid L$, correspond to the following relations on $G$ :

$$
R_{a \mid P}=\left\{(g, h):\left(\mathrm{p}^{g}\right)^{a}=\mathrm{p}^{h}\right\} \text { and } R_{a \mid L}=\left\{(g, h):\left(\mathrm{I}^{g}\right)^{a}=\mathrm{I}^{h}\right\} .
$$

By the choice of I incident with $\mathrm{p}$ and the fact that $G$ is a collineation group of $\Pi$, these two relations on $G$ coincide and equal $\{(g, h) \in G \times G: g a=h\}=R_{\rho(a)}$.

Suppose $a, b \in G$ and $f=\left(\mathrm{p}^{a}, I^{b}\right) \in P \times L$ is an ordinary flag. Since $G$ acts regularly on $P$ and on $L$, there is a unique flag in $f^{G} \subset F$ with point p. Consequently, $R_{f^{G}}$ is the table of values of the bijective function $a x \leftrightarrow b x: G \rightarrow G$. This function is $\lambda\left(a b^{-1}\right)$ because

$$
R_{f^{G}}=\{(a x, b x): x \in G\}=\left\{\left(y,\left(a b^{-1}\right)^{-1} y\right): y=a x \in G\right\}=R_{\lambda\left(a b^{-1}\right)} .
$$

Now $F$ is a disjoint union of $G$-orbits, each of which contains a unique flag having line I, so

$$
R_{F}=\bigcup_{d \in \Delta} R_{\lambda(d)}, \text { where } \Delta=\left\{d \in G:\left(\mathrm{p}^{d}, \mathrm{l}\right) \in F\right\} .
$$

In other words, for $h \in G$, the set of ordinary points incident with $\mathrm{I}^{h}$ is $\left\{\mathrm{p}^{d h}: d \in\right.$ $\Delta\}$, or simply that $\Delta h$ is the set of labels for points incident with $\mathrm{I}^{h}$.

Lemma 3. Suppose $a \in A$. Then

$$
R_{a} \circ R_{F}=R_{a \mid P} \circ R_{F}=R_{F} \circ R_{a \mid L}=R_{F} \circ R_{a},
$$

where $R_{1} \circ R_{2}:=\left\{(g, k) \in G \times G: \exists h \in G\right.$ so that $\left.(g, h) \in R_{1},(h, k) \in R_{2}\right\}$ is the composition of the relations $R_{1}$ and $R_{2}$.

Proof. The first and last equalities follow from the choice of $I$ incident with $\mathrm{p}$ as already noted. Take $(g, h) \in R_{a \mid P} \circ R_{F}$. By definition of $\circ,\left(\left(\mathrm{p}^{g}\right)^{a \mid P}, \mathrm{I}^{h}\right) \in F$. But $a$ is a collineation, so there is $s \in G$ such that $\left(\mathrm{p}^{g}, \mathrm{I}^{s}\right) \in F$ and $\mathrm{I}^{h}=\left(\mathrm{I}^{s}\right)^{a \mid L}$. Again, by definition of $\circ$, we have that $(g, h) \in R_{F} \circ R_{a \mid L}$.

\section{Planes of type I.4}

Finally, suppose $\Pi$ is of type I.4 and that the $(z, x y)$-transitive perspectivity group $Z$ is present. By Lemma 1, Lemma 2 and the symmetry of the Lenz-Barlotti figure, $Z$ acts on the ordinary points $P$ as a permutation group that commutes with $G$, and $G$ acts regularly on $P$. Also by Lemma 2 and by the symmetry of the Lenz-Barlotti figure, $Z$ is isomorphic to both $X$ and $Y$.

By yet another application of Theorem 2 and the synchronized choices of $\mathrm{p}$ and I, there is a monomorphism $\phi: Z \rightarrow G$ such that the point permutation $z_{P}$ and line permutation $z_{L}$ induced by $z \in Z$ are given by

$$
\mathrm{p}^{g} \rightarrow \mathrm{p}^{\phi(z)^{-1} g} \text { and } \mathrm{I}^{g} \rightarrow \mathrm{I}^{\phi(z)^{-1} g} \text { or, equivalently, } R_{z_{P}}=R_{\lambda(\phi(z))}=R_{z_{L}} \text { for } z \in Z \text {. }
$$

The action of $G$ on the z-axial lines is realized in the internal coset-geometry by right multiplication on $G_{\mathrm{pz}} \backslash G$. By definition, $Z$ acts on $P$ leaving each of the z-axial lines invariant and so $\lambda \circ \phi(Z)$ acts trivially on $G_{\mathrm{pz}} \backslash G$. This is only possible because $\lambda$ involves left multiplication in $G$, and it implies that $\phi(Z)=G_{\mathrm{pz}}$.

Take $z \in Z$ and use Lemma 3, with $a=z$, to rewrite equation (1) by applying equation (2) to obtain: $R_{\lambda(\phi(z))} \circ R_{F}=R_{F} \circ R_{\lambda(\phi(z))}$, for all $z \in Z$. This is 
equivalent to an equality of sets of permutations in $\operatorname{Sym}(G)$ :

$$
\bigcup_{d \in \Delta} \lambda(\phi(z)) \lambda(d)=\lambda(\phi(z))\left[\bigcup_{d \in \Delta} \lambda(d)\right]=\left[\bigcup_{d \in \Delta} \lambda(d)\right] \lambda(\phi(z))=\bigcup_{d \in \Delta} \lambda(d) \lambda(\phi(z)) .
$$

But $\lambda$ is a group isomorphism and we have just shown that $\phi(Z)=G_{p z}$, so

$$
h \Delta=\Delta h \text { for all } h \in H=G_{\mathrm{pz}} .
$$

The (z, xy)-perspectivity group, $Z$, fixes w, so the $Z$-orbit of I contains no ordinary points on $z w$. Therefore, no ordinary point on $z w$ is labeled by an element of $\bigcup_{h \in H} h \Delta$.

By choice of $\mathrm{w}, \mathrm{w}^{h} \neq \mathrm{w}$ for some $h \in H$. Therefore, the ordinary points labeled by $\bigcup_{h \in H} \Delta h$ include all ordinary points on $\mathrm{I}^{h}$. In particular, $\mathrm{I}^{h} \mathrm{zW}$ is labeled by an element of $G$ in $\bigcup_{h \in H} \Delta h$ (see Figure 1). Now, by the choice of I (at the end of the second paragraph of the preceding section), this contradicts equation (3) and completes the proof.

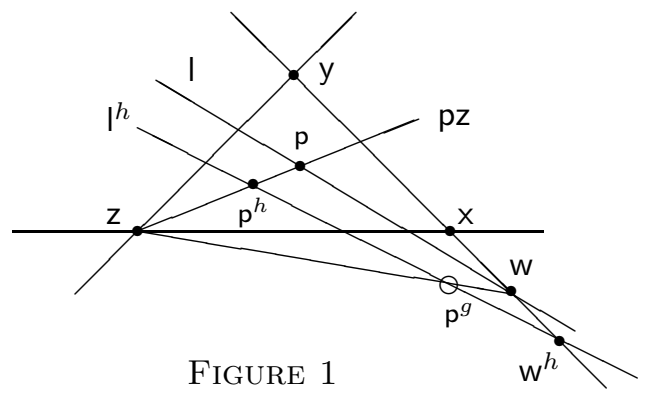

\section{REFERENCES}

1. D. Ghinelli and D. Jungnickel: On finite projective planes in Lenz-Barlotti class at least I.3. Advances in Geometry 2003, suppl., S28-S48. MR2028386 (2005a:51011)

2. M. Hall: The Theory of Groups, Macmillan, New York, 1959. MR0103215 (21:1996)

3. D.R. Hughes: Partial difference sets. Amer. J. Math. 78 (1956), 650-674. MR0084785 (18:921b)

4. C. Jordan: Traité des substitutions et des équations algébriques. Gauthiers-Villars, Paris, 1870.

5. W.M. Kantor: Projective planes of type I-4. Geometrica Ded. 3 (1974), 335-346. MR0383233 $(52: 4114)$

6. W.M. Kantor and M.D. Pankin: Commutativity in finite planes of type I-4. Arch. Math. (Basel) 23 (1972), 544-547. MR0320883 (47:9416)

7. H. Naumann, Stufen der Begründung der ebenen affinen Geometrie. Math. Zeit. 60 (1954), 120-141. MR0063058(16:64c)

8. H. Wielandt: Finite Permutation Groups, Academic Press, New York, 1964. MR0183775 (32:1252)

Department of Mathematics, Colorado State University, Fort Collins, Colorado 80523

Department of Mathematics, Armstrong Atlantic State University, Savannah, GeorGIA 31419

Current address: 3063 West Chapman Avenue, Orange, California 92868 